\title{
Launch Vehicle Propulsion Design with Multiple Selection Criteria
}

\author{
Joey D. Shelton* \\ NASA, George C. Marshall Space Flight Center, Huntsville Alabama \\ Robert A. Frederick ${ }^{\dagger}$ \\ The University of Alabama in Huntsville, Huntsville, Alabama
}

Alan. W. Wilhite $\ddagger$

The Georgia Institute of Technology, Atlanta, Georgia

The approach and techniques described herein define an optimization and evaluation approach for a liquid hydrogen/liquid oxygen single-stage-to-orbit system. The method uses Monte Carlo simulations, genetic algorithm solvers, a propulsion thermo-chemical code, power series regression curves for historical data, and statistical models in order to optimize a vehicie system. The sysiem, inciuding parameters for engine chamiber pressuie, àrea râtiou, ânủ oxidizer/fuel ratio, was modeled and optimized to determine the best design for seven separate design weight and cost cases by varying design and technology parameters. Significant model results show that a $53 \%$ increase in Design, Development, Test and Evaluation cost results in a 67\% reduction in Gross Liftoff Weight. Other key findings show the sensitivity of propulsion parameters, technology factors, and cost factors and how these parameters differ when cost and weight are optimized separately. Each of the three key propulsion parameters; chamber pressure, area ratio, and oxidizer/fuel ratio, are optimized in the seven design cases and results are plotted to show impacts to engine mass and overall vehicle mass.

\footnotetext{
${ }^{*}$ Systems Engineer, Space Transportation Programs and Projects Office, George C. Marshall Space Flight Center/NP20, AIAA Member.

$\dagger$ Associate Director, UAH Propulsion Research Center, 5000 Technology Drive, and AIAA Associate Fellow

¥ Langley Distinguished Professor, GIT Center for Aerospace Systems Analysis, National Institute of Aerospace, Hampton, VA. AIAA Associate Fellow 


\section{Nomenclature}
$\mathrm{AR}=$ Area Ratio
$\mathrm{CLO}=$. Closed Loop Optimization
GLOW $=$ Gross Liftoff Weight
ISP $=$ Specific Impulse
$\mathrm{OF}=$ Oxidizer/Fuel
$P_{c} \quad=$ Chamber Pressure
SSTO $=\quad$ Single Stage to Orbit
$\mathrm{T}=$ Thrust
Twi $=\quad$ Thrust to Weight Ratio

\section{Introduction}

$T_{\text {way. Propulsion variables such as specific impulse (ISP), engine mass, gross liftoff weight (GLOW), nozzle area ratio }}^{\mathrm{O} \text { date, few modeling tools have attempted to incorporate key propulsion system variables in a totally iterative and optimum }}$ (AR), chamber pressure (Pc), initial thrust-to-weight ratio (Twi), and oxidizer/fuel (OF) ratio, have not been fully integrated into a model for closed loop analysis of launch vehicle concepts. Performance characteristics for propulsion optimization are measured by weight, reliability and cost of operations. The primary goals of the optimization studies herein are based upon the weight reduction in the propulsion system but also focus on minimizing system cost. This study focuses on rocket systems that utilize liquid hydrogen fuel and liquid oxygen oxidizer. A major factor in minimizing overall systems development cost is accomplished by optimizing propulsion system components using historical engine data combined with a propulsion thermochemical code, and optimization tools/techniques.

The model process flow defined is Figure 1 is formed for a single stage to orbit (SSTO) system using liquid oxygen (LOX) and liquid hydrogen (LH2) and focuses on minimization of key parameters relating to vehicle weight and cost. Key performance measures are defined for GLOW minimization, Dry Weight minimization, Dry Weight with Margin minimization, Design, Development, Test and Evaluation cost minimization, Production cost minimization, Operations cost minimization, and Life Cycle cost minimization. The approach uses a combination of historical data for weight relationships, Monte Carlo simulations for uncertainty analysis, technology factors and cost influence factors for trading weight savings for cost savings, thermochemical analysis, and genetic algorithm solvers for concept optimization. Analysis for each design cases results in optimized parameters for Pc, AR, OF ratio, Twi, mass ratio (MR), technology factors (TFs), and cost influence factors (CIFs). Monte 
Carlo simulation uncertainty is used to determine the performance measures for cost and/or weight based upon lognormal distribution functions at a $95 \%$ uncertainty level. The results of the study reflect the improvements in methodology and the practical use of the tool to measure propulsion parameter sensitivity when compared to the key performance measures.

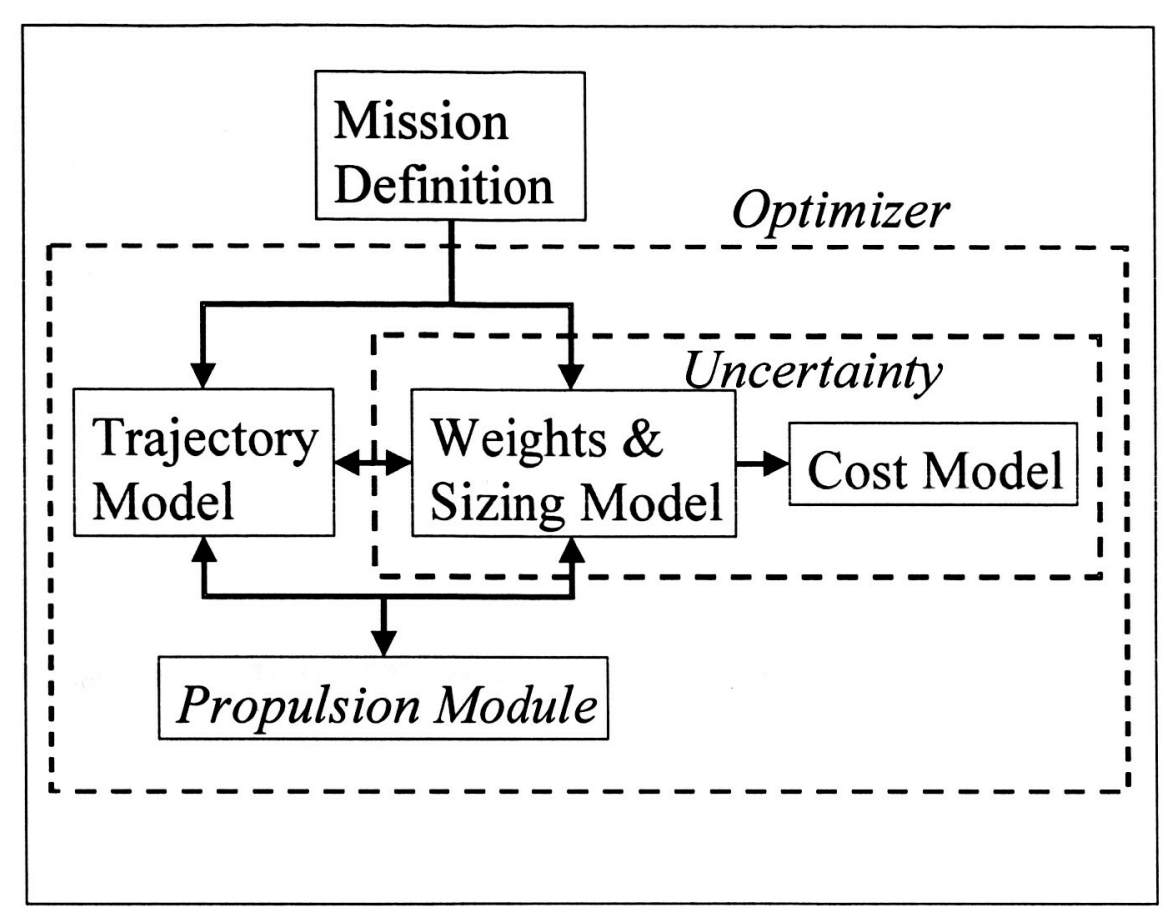

Figure 1, Model Process Flow

\section{Approach}

The optimization of the integrated vehicle SSTO model using rocket engines focused on several key areas. The first is the overall optimization of the vehicle Twi, engine Pc, engine AR, engine OF Ratio, and TFs for TPS, Autonomous Flight Control, Wing, Tail, LH2 Tank, LOX Tank, Basic Structure, Landing Gear, Engine Accessories, MPS, Engine, and Flight Autonomy. The TFs are used to vary the system weight by improved technology but can also result in a DDTE cost increase/decrease due to the time and effort expended in developing an advanced technology. The combination of the propulsion variables and TFs used to define the optimum system with respect to the following Figures of Merit (FOMs): 1) Minimum GLOW, 2) Minimum Dry Weight, 3) Minimum Dry Weight with Margin, 4) Minimum DDTE Cost, 5) Minimum Production Cost, 6) Minimum Operations Cost, 7) and Minimum Life Cycle Cost (LLC). A table of optimization results is calculated for each of the seven different FOMs defined above. The vehicle MR, Main Engine Throat Diameter (D*), Vacuum Specific Impulse (ISPvac), Seal Level Specific Impulse (ISPsl), per engine Thrust (T), and per engine weight estimation are defined for each of the seven optimal results and is derived for each FOM case. These levels will vary in each of the seven scenarios due to the fact that the optimization is focused 
on key FOMs that drive the final optimized propulsion parameters and TFs toward different solutions or goals. In some cases the solutions may be near identical because of the similarity in the parameter being optimized. A comparison is made between the model output and the SSME main engine design parameters. The information is defined in order to validate the model and show that the solution proposed by the integrated model closely aligns with the current best practices in engine system design used by NASA.

\section{A. Reference Vehicle}

The first step was to define a concept for a rocket propelled SSTO vehicle in order to build the optimization model. The concept vehicle chosen is based upon NASA CR2866 [1]. The vehicle utilizes LOX as the oxidizer and LH2 fuel and uses fixed nozzle engines to propel the vehicle to orbit. The concept incorporates improved technologies for enhanced performance as well as using historical data to produce projections for future technology upgrades in the areas of material science for composite and metal matrix composites, rocket engine efficiency, and lighter and more thermally resistant protection system for the vehicle exterior. For the analysis of the launch vehicle and the launch vehicle propulsion system, a model of the entire system was developed using equations for rocket propulsion parameters, trajectory profiles, weights and sizing, cost and economics, and historical data.

The approach required to model a system of fixed nozzle engines was significantly less difficult. The important aspects of this computer modeling approach is to show how the model can be developed and how the tools can be used to design, analyze and optimize the system, not how to define a complex system. The concept vehicle chosen requires the following specification parameters; 1) Vertical Takeoff, 2) Dual-Engine Mode, 3) 500 Mission Lifetime, 4) 65,000 lb Payload, 5) 28.5 degree Launch Inclination from the NASA John F. Kennedy Space Center, 6) and $50 \mathrm{nmi}$ Orbit [1]. The concept vehicle is shown here in Figure 2. 


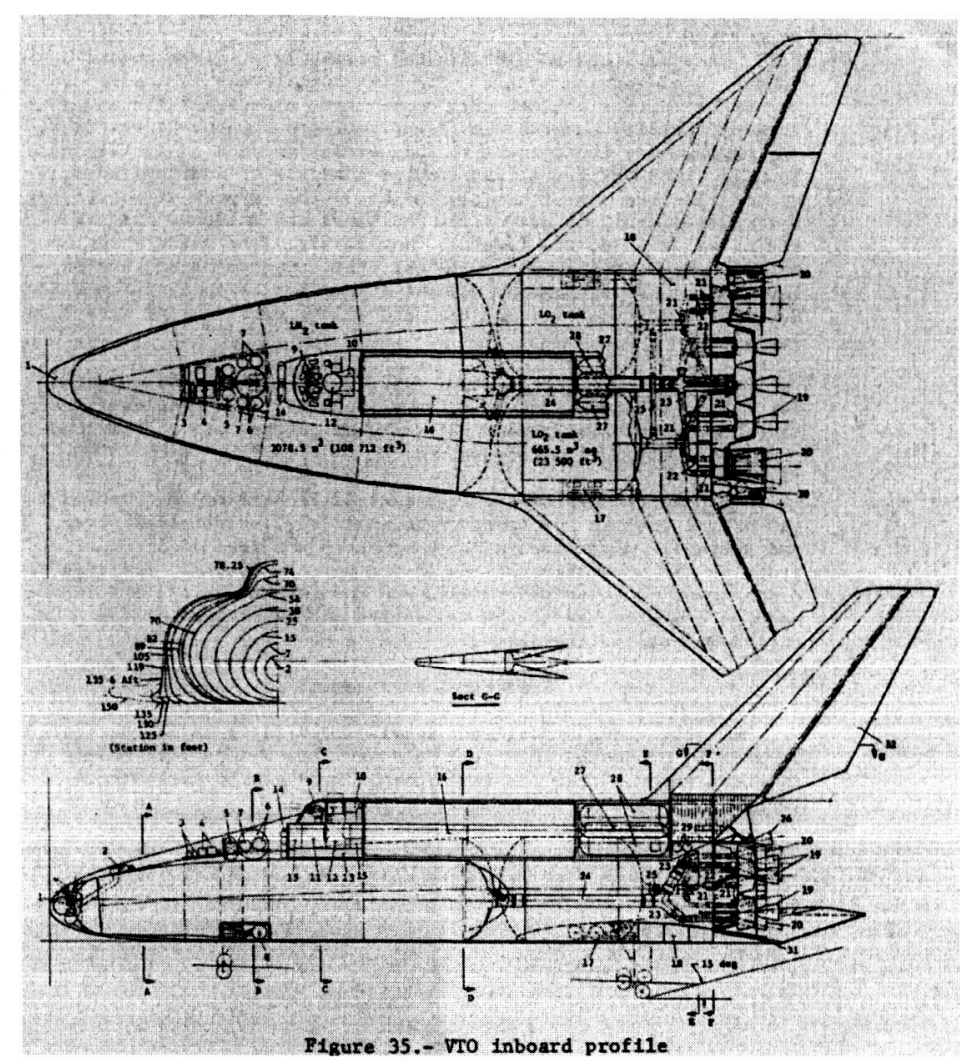

Figure 2, SSTO Concept Vehicle [1]

\section{B. Simulation Architecture}

The trajectory model uses the rocket equation to formulate a MR for the vehicle system. This module also includes a pressure, temperature and density parameter calculation based upon altitude. The program uses input values for $\Delta \mathrm{V}$ required, orbit inclination, orbit height, and drag reduction percentage. These values are set and determined based upon the concepts for a specified mission and will not change during the optimization of the design process. The module also takes Twi, ARs, Pc, OF Ratio, Delta ISP, Engine Throat Area divided by the Vacuum Thrust (A*/Tvac), and ISPvac and uses these values to determine a value for MR of the vehicle for the given reference conditions. The Twi, AR, Pc, OF Ratio, Delta ISP, and A*/Tvac are parameters input into the trajectory program by the user.

The value(s) for ISPvac are inputs from the Propulsion Module. The propulsion module will define a value for ISPvac based upon the Pc, AR, and OF Ratio optimization outputs from the Trajectory Model. This relationship is described in more detail during the discussion of the Propulsion Model in Section C. The Trajectory Model has the option for defining dual engine systems and the ability to specify the percentage of thrust being provided by engine set 1 versus the total (T1_T), and having an engine set 2 which allows for an expandable nozzle to be extended at some point during the trajectory. The value for this term is 
a percentage of the trajectory and is defined as the Area Ratio Transition (ART). The significance of the adjustable nozzle is to allow for greater area ratios later in flight without having the negative pressure benefits of atmospheric pressure that occurs earlier in the trajectory. Therefore, the ART will be later in the flight when atmospheric pressure is low. This is mentioned for illustration as a model capability. This system being described herein does not use dual engine and expandable nozzle functions.

The rocket equation is based upon the calculation of a $\Delta V$ by using the vehicles ISP, MR, Drag (D), Thrust, Weight (W), and launch angle $(\gamma)$. When solving for $\Delta \mathrm{V}$, the form of the equation (1.1) is shown here,

$$
\Delta V=g o * i S P \ln (M R)(1-D / T-W / T \sin \gamma)
$$

The Trajectory program solves for the vehicle MR as the primary output. The output also includes Time-to-Orbit, Final Velocity, Final Altitude. The output values of Final Velocity and Final Altitude must match the DRM inputs mentioned earlier. The rocket equation (1.1) is solved for MR and is shown in equation (1.2),

$$
M R=\Delta V /(I S P *(1-D / T-W t / T * \sin \gamma)
$$

The input parameters for Twi, AR, Pc and OF ratio are modified during the analysis of the system by using the GA optimization Palisades Tool Evolver [2]. By choosing to minimize the Overall Vehicle Dry Weight, the program will define the best possible configuration for reduced weight by changing the input parameters. The Weights and Sizing Model and the Propulsion Module use these parameters as inputs.

\section{Propulsion}

The Propulsion Module resides as a worksheet within the Weights and Sizing Model. The Propulsion Module utilizes parameters calculated using Cequel [3]. Cequel uses the minimization of Gibbs Free Energy and provides combustion process outputs based upon rocket inputs. Key propulsion parameters inputs to Cequel include Pc, AR, and OF ratio. These three parameters are input into Cequel after having been optimized in the Trajectory Model. The model uses only the ISPsl, ISPvac, thrust coefficient (Cf), and characteristic exhaust velocity (Cstar) outputs from Cequel. The outputs are integrated with the Weights and Sizing, and Trajectory models to determine the overall vehicle weights and performance output.

The launch vehicle's required sea level thrust (Tsl) is determined by multiplying the GLOW times the Twi as shown in equation (1.3), 


$$
T_{s l}=G L O W * T w i
$$

The value for Twi is also used during the Genetic Algorithm (GA) optimization procedure. The Propulsion Module utilizes Sea Level Thrust (Tsl) and the value for ISPsl, to calculate the rate of change for the engine weight (Wdot). The value of Wdot is defined as the rate of change in overall vehicle weight and varies with changes in GLOW and/or ISPsl, as shown in equation (1.4),

$$
W_{d o t}=\frac{G L O W}{I S P_{s l}}
$$

The value for ISPsl is an output from the Cequel code. Any change to the vehicle model that impacts the value of these two parameters (GLOW and ISPsl) has an impact on the value of Wdot. This is an important point and should not be lost when understanding how propulsion model functions as a closed loop system. The significance lies in the understanding that when a parameter changes it has impact to other values in the model. Understanding this relationship and how to model it is the most significant accomplishment of this problem.

The value for Tvac is defined based upon the Wdot, calculated in equation (1.4) above, multiplied times the Cequel output for ISPvac. The relationship between Tvac and Tsl is established using Wdot because the propellant flow rate is the same regardless whether the value is determined using sea level conditions or vacuum conditions. This relationship is shown here in equation (1.5) and gives the Tvac based upon thermo-chemical output for ISPvac,

$$
T_{v a c}=W_{d o t}^{*} I S P_{v a c}
$$

The engine design parameter for Combustion Efficiency is $96 \%$, and is based upon historical engine system and studies performed by the NASA-MSFC Transportation Directorate organization during the development of the NASA MSFC Pstar tool [4]. The efficiency level reduces the Cequel output for ISPvac and ISPsl by $4 \%$. The user can modify the efficiency if level is deemed to high or low for a particular system. Any values for engine design parameters, including Trajectory Model optimization parameters, are using the reduced level of ISP due to efficiency. The ISP value is an important component in the Trajectory Model's determination of the vehicle MR, as shown in equation (1.1). An important integration point is that the value for ISP is a result from the design inputs being used to size the system and to define the propulsion system. 
The Propulsion Module also provides the correlation of engine parameters to the engine weight. The engine weight is determined using a combined Rocketdyne Power Balance, U.S. Air Force, NASA Langley approach and historical engine data to develop a curve that relates engine weight to the value found when multiplying OF Ratio, AR and Pc together. The four methods are averaged together to give an engine weight based upon these methods. The Power Balance and U.S. Air Force methods make use of the nozzle geometry model in order to determine nozzle weight. The nozzle weight is determined using engine AR, $A^{*}$ and coefficients for unit weights of the material used in the design of the nozzle.

The combined parameters, shown in Figure 3-3, define variables used to determine Engine Thrust required such as, $A^{*}$ and mass flow rate (mdot). The equations (1.6) and (1.7) show how Sutton [5] defined A* and subsequently throat diameter $\left(\mathrm{D}^{*}\right)$ from the propuision parameters,

$$
A^{*}=\frac{T}{P_{c}{ }^{*} C_{f}},
$$

and

$$
D^{*}=\sqrt{\frac{4^{*} A^{*}}{\pi}} .
$$

The key to determining the engine geometry and weight is establishing the value for several secondary parameters. The value for mdot of the engine is defined by Sutton [5] and is illustrated in equation (1.8),

$$
m_{d o t}=\frac{P_{c}^{*} A^{*}}{C_{s t a r}}
$$

Where Cstar is define in the thermo-chemical output calculation using Cequel. The results for mdot will be used in the determination of the engine mass.

Engine mass is a function of the calculation techniques defined by the U.S. Air Force [6], NASA Langley approach [7], Rocketdyne Power Balance [8] and historical data curves for the relationship between OF Ratio*Pc*AR and engine mass. The resulting predicted engine mass is determined using the mass average of each of these four approaches. The engine mass relationship is important to the overall model because of the significance in the engine mass as a percentage of the overall GLOW. Equations (1.9) and (1.10) show which parameters are used to determine the nozzle mass and then overall engine mass. An important point is to note is that Cequel defines both $\mathrm{Cf}$ and Cstar and provides a solution approach with the precision that only the thermo-chemical solution can provide. 


$$
\text { NozzleMass }=f\left(A^{*}, A R\right),
$$

and

$$
\text { EngineMass }=f\left(m_{d o t}, C_{f}, A R, P_{c}, \text { Thrust }, \text { OFRatio, } I S P_{s l}\right)
$$

\section{Weights and Sizing}

The first phase of the model development addressed the building of a core weights and sizing model of the concept vehicle. This module utilizes a modified version of a Microsoft Excel model called Launch Vehicle Sizer and Synthesis (LVSS) [9]. The LVSS component is made up of several Microsoft Excel worksheets that share data.

The initial inputs are dedicated to input variable definition for the vehicle MR, Twi, payload weight, delta-velocity requirements, engine ISPs, and ARs. The Propellant worksheet is used to formulate a propellant module for determining tank volumes and weights based upon inputs for density of fuel, payload volume and body volume. The Propulsion worksheet is used to determine the engine masses based upon the ISP, area ratio, engine compilation, and thrust level needed. The Weight Equations worksheet is dedicated to determining the vehicle component weights using historical data and technology factors defined for each component. The Sizing worksheet provides the outputs for the vehicle sizing based upon inputs from the previous sheets.

\section{Cost and Economics}

The cost and economics model is derived based upon the NASA cost projection methodology [10]. The model is developed using the component weights of previous systems, and plotting the cost of the component versus the weight of the component. The resulting regression curves are used to project future cost for a particular system. The CIFs that result from using technology factors are used to calculate the cost of a component DDTE. The higher technology factors (TFs) will decrease the weight of component or increase the effectiveness of a component, but will almost always increase the cost of development. The factors, both TFs and CIFs, are model design and analysis parameters that will change as technology evolves and also as the ability to efficiently manufacture or develop a design is enhanced or minimized. A Boolean code is used to related 1, 2 or 3 with the desired TF and CIF.

The uncertainty surrounding the DDTE cost is high due to the large number or data points used in the fitting the historical data to a regression curve. The approach uses a Log Normal Monte Carlo Uncertainty based upon data standard errors (SEs). All of the subsystem components have SE's that are used in determining the overall DDTE cost uncertainty value. In addition to the subsystems, the NAFCOM model also applies standard errors for the RCS, and OMS. 


\section{E. Simulation Operation}

The capability of the Evolver optimizer tool [2] enables the analyst to define a set or predetermined value for TFs and key propulsion parameters if the technology is not available or if the program or project agrees to use either a new technology or established technology. This optional feature allows the designer the flexibility to also define any of the seven FOMs at a particular value or add a constraint to the optimization. An example would be to include DDTE cost as an independent variable (CAIV) and optimize the vehicle with this cost value set as a constraint. The model will allow any of the other 7 FOMs to have set values in order to meet a congressional, agency, or industry mandate. The same ability to set pre-defined levels for FOMs or other variables also applies to the propulsion system if for example the use of existing engines was mandated.

The second capability of the model is to show how each of the key engine parameters, Pc, OF Ratio, and AR are optimized against the FOMs and measure the changes to the system when the optimized value is not met. The sensitivity of these key propulsion parameters is extremely important to the system designer. The model provides the results that are used to show how changes in each propulsion parameter effects the 7 FOMs mentioned earlier. Understanding the impacts that a parameter has on the overall system is crucial to vehicle system design. Design decisions should never be made without understanding the full impacts of upon the overall system.

The Figures 3 and 4 illustrate how each of the given propulsion parameters is used as inputs to determine nozzle mass, engine mass, propellant system mass and vehicle MR and illustrates other uses for the propulsion parameters. An overall system flow diagram is shown in Figure 5. Each section of the model has input and outputs that feed the system and form the closed loop optimization methodology so desired. The overall schematic shows how mission requirements are fed to the system and how each model component (LVSS-Propulsion Module, Trajectory, Cost and Economics) interacts and what key variables are passed between the models. 


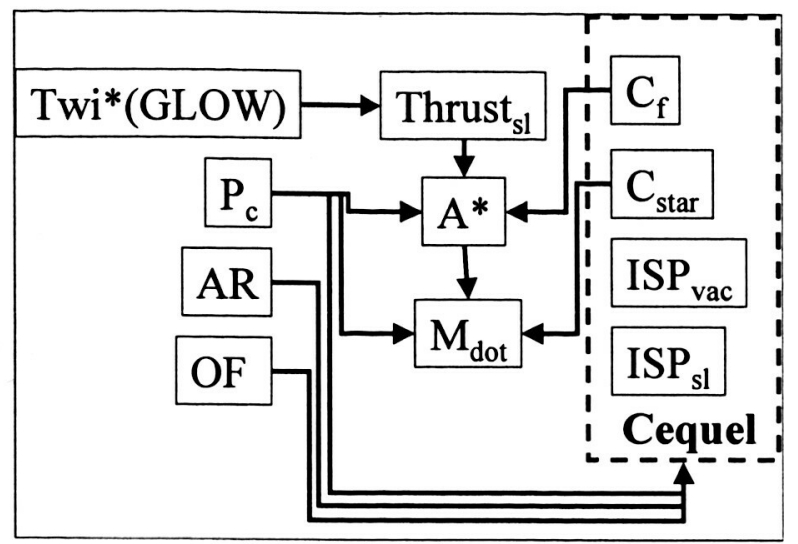

Figure 3, System Propulsion Parameters

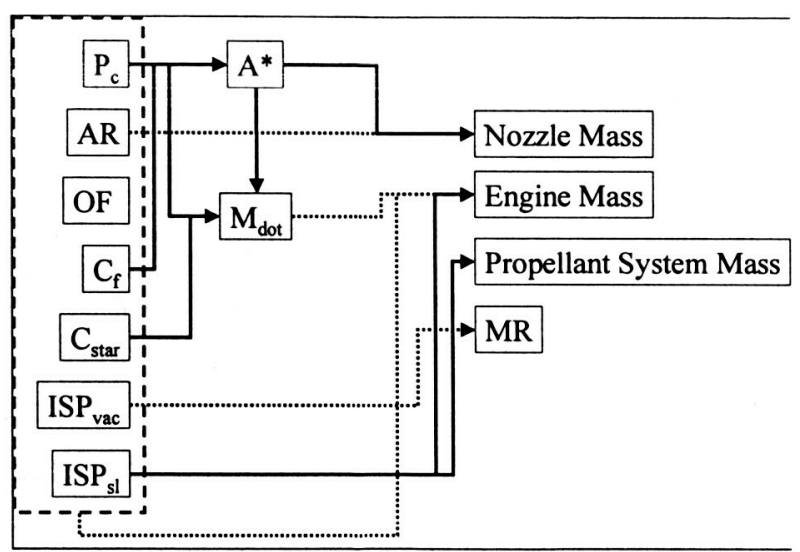

Figure 4, Model Process Diagram 


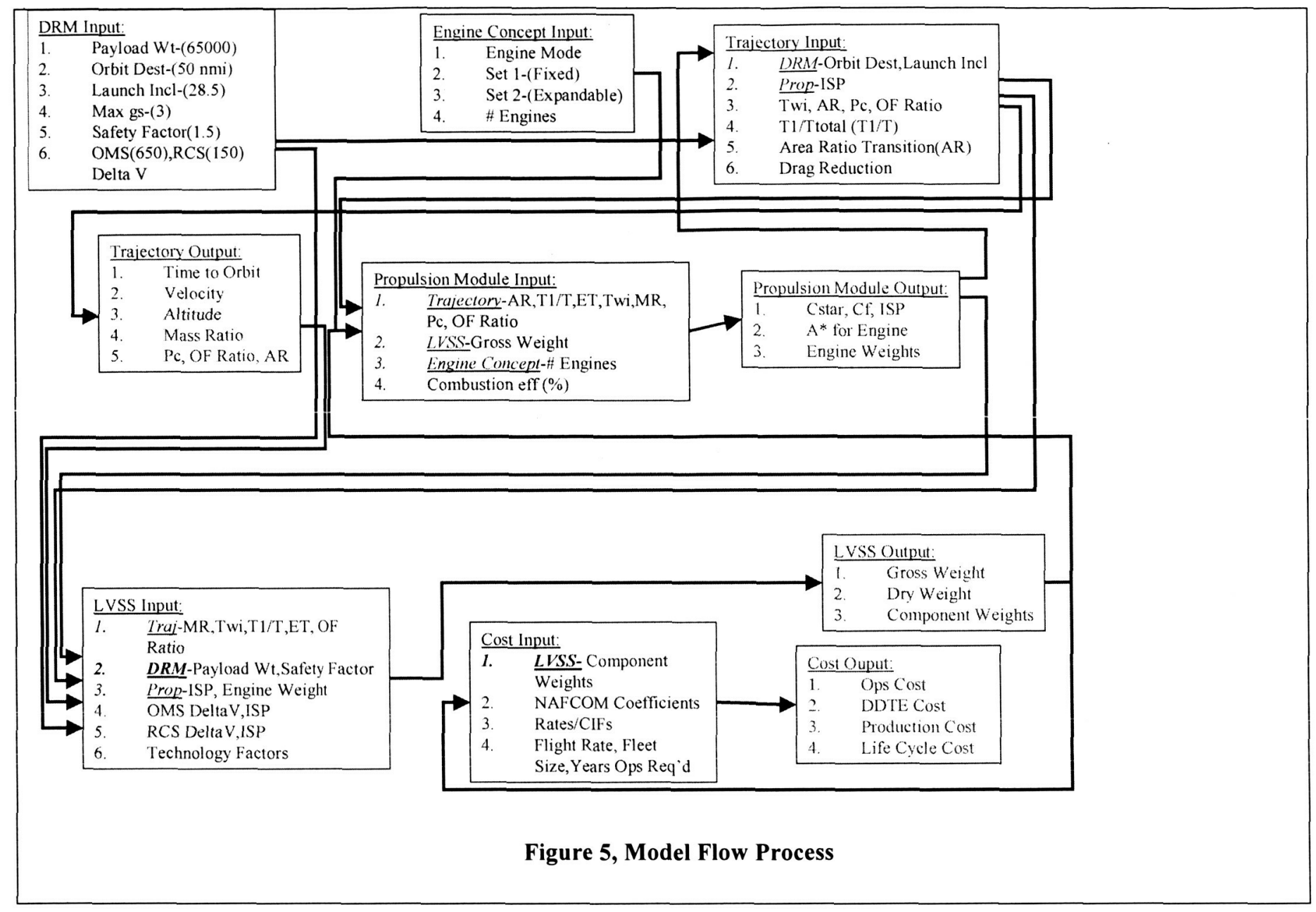

The approach utilizes uncertainty principles in order to bind the final outputs with a measure of goodness or certainty. In the case of the LVSS and the DDTE cost, the historical data curves were used to develop estimates for the component vehicle weights and regression curves defined the $+/$ - regression range for each of the curves. 


\section{Results and Discussion}

The optimal vehicle design defined herein is based upon the minimization of the seven key FOMs. For each of the seven cases, the values propulsion parameters are defined using the Palisades Evolver optimization tool. Secondary engine design parameters are defined as well in order that the engine system specification could be written from the model output. The range of TFs and corresponding CIFs are based upon the Boolean code for technologies 1,2 or 3 . The Tables 1 and 2 show the results of the GA Evolver Tools results for the optimal vehicle design. The three cases of weight minimization are shown in Table 1; Minimum GLOW, Minimum Dry Weight, Minimum Dry Weight with Margin, and the four cases where cost is minimized are shown in Table 2; Minimum DDTE Cost, Minimum Production Cost, Minimum Operations Cost, and Minimum Life Cycle Cost (LLC). The relationship between the weight components shows an absolute correspondence between the Dry Weight and the Dry Weight with Margin. The cost numbers were slightly different between the GLOW and Dry Weight, with the upfront cost (DDTE/Production) of the GLOW optimized solution being higher. The downstream cost of the GLOW optimized system was slightly lower that the Dry Weight optimized solution. The Pc and AR were nominally unchanged but the OF Ratio and Twi were significantly different between the Dry Weight and GLOW cases. The OF Ratio for Dry Weight optimization is focused on tank weight, while the GLOW optimized solution is focused on lower fuel weight. Flight Autonomy is the only TF that differed between the multiple runs of the 2 weight optimization cases. The Boolean code for the Flight Autonomy relates 1-Shuttle Like, 2-Semi Airplane Like, and 3-Airplane Like. The remaining TFs can be understood by referencing Table 3. The table explains the Boolean Code link to the material property for each, either 1,2 or 3. 


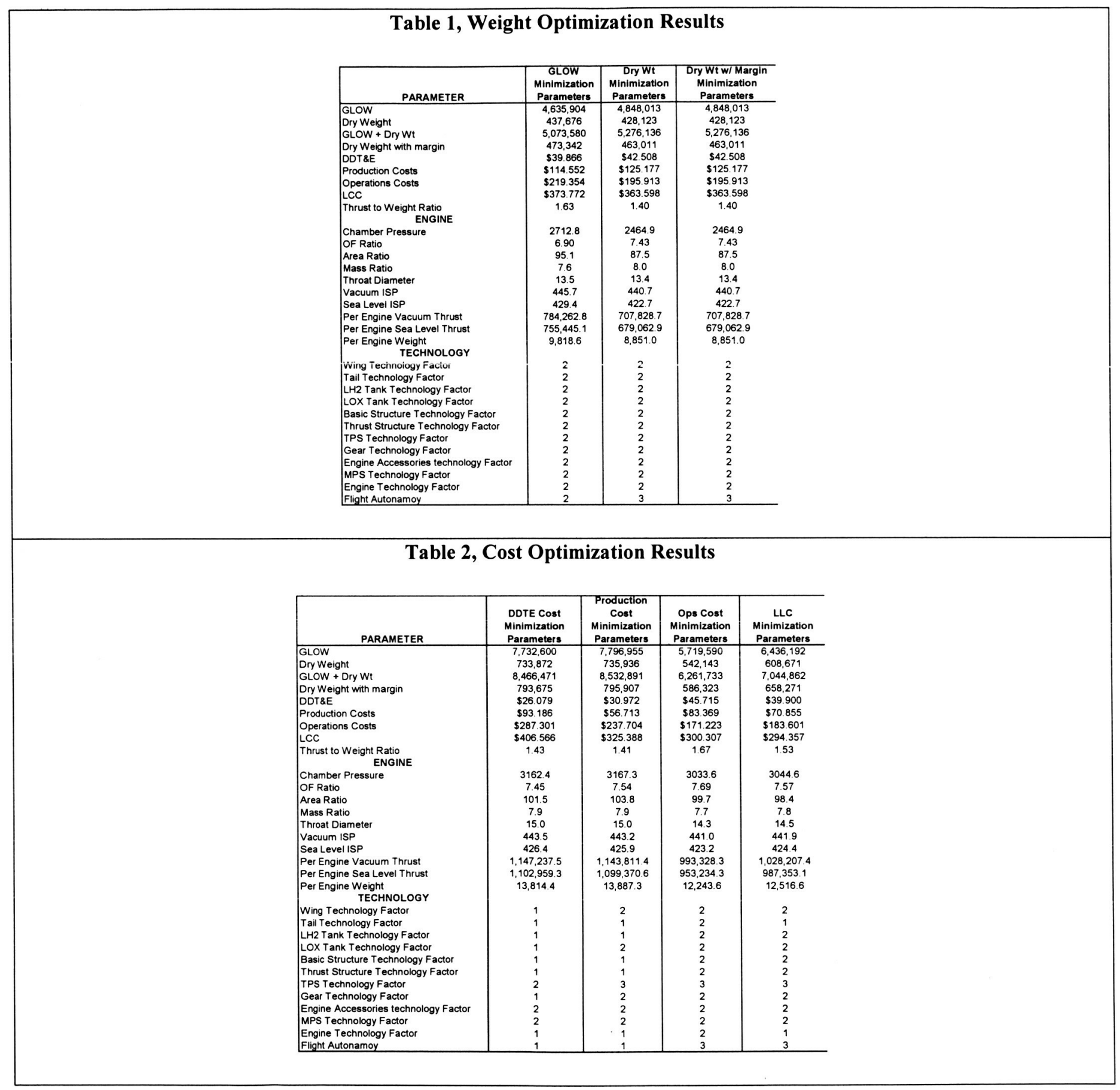


Table 3, Boolean Code Logic

\begin{tabular}{|l|r|r|l|r|r|r}
\hline Parameter & $\begin{array}{l}\text { Boolean } \\
\text { Code }\end{array}$ & Factor & Material & Code 1 & Code 2 & Code 3 \\
\hline Wing Technology Factor & 3 & 0.869 & MMC & 1.000 & 0.724 & 0.869 \\
Tail Technology Factor & 3 & 0.970 & MMC & 1.000 & 0.842 & 0.970 \\
LH2 Tank Technology Factor & 3 & 0.900 & MMC & 1.000 & 0.750 & 0.900 \\
LOX Tank Technology Factor & 2 & 0.700 & Composite & 1.000 & 0.700 & 0.840 \\
Basic Structure Technology Factor & 2 & 0.700 & Composite & 1.000 & 0.700 & 0.840 \\
Thrust Structure Technology Factor & 2 & 0.740 & Composite & 1.000 & 0.740 & 0.888 \\
Gear Technology Factor & 1 & 1.000 & Aluminum & 1.000 & 0.781 & 0.937 \\
Engine Accessories technology Factor & 1 & 1.000 & Aluminum & 1.000 & 0.891 & 1.070 \\
Wing CIF (Cost influence Factor) & 3 & 10.000 & MMC & 1.000 & 2.100 & 10.000 \\
Tail CIF & 3 & 10.000 & MMC & 1.000 & 2.100 & 10.000 \\
LH2 tank CIF & 3 & 10.000 & MMC & 1.000 & 2.100 & 10.000 \\
LOX tank CIF & 2 & 2.100 & Composite & 1.000 & 2.100 & 10.000 \\
Body CIF & 2 & 2.100 & Composite & 1.000 & 2.100 & 10.000 \\
Basic structure CIF & 2 & 2.100 & Composite & 1.000 & 2.100 & 10.000 \\
Thrust Structure CIF & 2 & 2.100 & Composite & 1.000 & 2.100 & 10.000 \\
Landing Gear CIF & 1 & 1.000 & Aluminum & 1.000 & 1.800 & 10.000 \\
\hline
\end{tabular}

The optimization parameters for the cost cases are shown in Table 2. The propulsion parameters are closely tied together for each of the 4 cost optimization cases. The only exception is the Twi value. The Twi for the minimum DDTE and Production cost is lower than the Twi for the minimum Operations and LCC cost. The significant difference for the cost cases is in the Boolean Codes $(1,2$ or 3$)$ for each of the 4 cases. This is logical because the TFs and more importantly the CIFs have a significant impact of cost due to the fact the CIFs are multiplying factors in the cost of each component.

The most significant differences between the weight parameters and cost parameters in Tables 1 and 2 are the TFs and corresponding CIFs. For the weight minimization cases, the TFs are driven to a Boolean value of two (2) and the DDTE cost minimization case in generally equal to one (1). These results are expected because the weight savings for the Boolean value of two (2) represents a weight savings over current baseline technologies but represent a cost increase by a multiple of 2.1 times the cost for current technology for a Boolean value of one (1). The GLOW, Dry Weight and Dry Weight with Margin reflect near identical TFs based upon the fact that the technologies defined in the database by Boolean two (2) represent a multiple for component weights that is less than one. The production, operations and life cycle cost reflect a wider range of TF and CIF possibilities. This is true because the most significant cost impact for new technologies is absorbed by the DDTE cost and will in some cases result in cost savings during the other cost phases of the program. In essence, increased cost incurred during the DDTE phase results in cost savings for the vehicle production, operations and life cycle.

The propulsion design parameters shown in Tables 1 and 2 vary based upon the specific minimization case. The chamber pressure influence follows the same trend for the GLOW, Dry Weight with Margin and Dry Weight minimization cases. The chamber pressure is slightly higher for the GLOW minimization case. This is due to the fact that the ISP level is higher at increased chamber pressure and thus vehicle requires less fuel. The Dry Weight minimization chamber pressure is lower due in 
great part to the sole focus on reducing engine mass. The lower the chamber pressure results in smaller engine mass. At high chamber pressures the chamber size and weight, lines and valves, and support structure must be more robust and drives the engine mass up. The cost optimization cases define the chamber pressure level at approximately 3100 psi. This level is approximately the level for the current SSME block design.

The area ratio for the various cases follows a similar patter to the chamber pressure described earlier. The area ratio is lowest for the Dry Weight minimization case because smaller area ratios result in a lighter engine nozzle. But the ratio must be high enough to enable the appropriate ISP. The GLOW case defines a slightly higher area ratio that is due in part to the increased specific impuise for the higher area ratio. The area ratio for the cost minimization cases is nearly identical and is slightly higher than the weight cases. The higher area ratio for the cost cases is driven by the increased specific impulse but is not impacted as severely by the magnitude of increased weight due to increasing the area ratio.

The optimized OF ratio is defined for each of the 7 minimization cases. The value for OF ratio is driven to an optimized solution for the GLOW case based upon decreased engine propellant mass. The OF ratio for the Dry Weight minimization case is higher because this ratio impacts the propellant tank volume only. So in essence, the optimized value for the OF ratio is most impacted by the propellant density and propellant volume. The OF ratio for the cost minimization cases is in the range similar to the GLOW minimization case. The ratio value for the DDTE cost minimization and the Dry Weight minimization cases are identical due to the correlation between the component weight relationships within the DDTE cost estimates.

The optimization cases, including propulsion parameters, are shown for OF Ratio, Pc and AR. OF Ratio has a significant impact on the overall weight of the vehicle and vehicle components due to the large percentage of the GLOW that is dedicated to fuel and oxidizer. The results from varying the Pc and AR prove that these parameters have a much lower impact on the GLOW and Dry Weight than the OF Ratio. The impacts of each of these parameters on the overall engine mass are shown for an incremental range of values. The results in subsequent sections show how each of the propulsion parameters will affect the vehicle and how the vehicle will be impacted when a propulsion parameter is changed to a value that is different than the optimum value.

OF ratio effects on vehicle Dry Weight, GLOW, ISPvac, propellant volume, propellant weight, propellant density and engine mass are defined in Figures 6 to 9. The program includes an Excel macro that increments the OF Ratio from 5.0 to 8.0 and shows the results on each parameter. The incremental values for the OF Ratio are run against overall vehicle optimization case 
for Dry Weight minimization. The data for the OF Ratio that corresponds to the minimum Dry Weight, minimum GLOW, maximum ISPvac, minimum propellant volume, minimum propellant weight, and minimum engine mass are found using the Excel VLOOKUP function search. The search returns the value for the OF Ratio that results in the minimum or maximum of the case being analyzed.

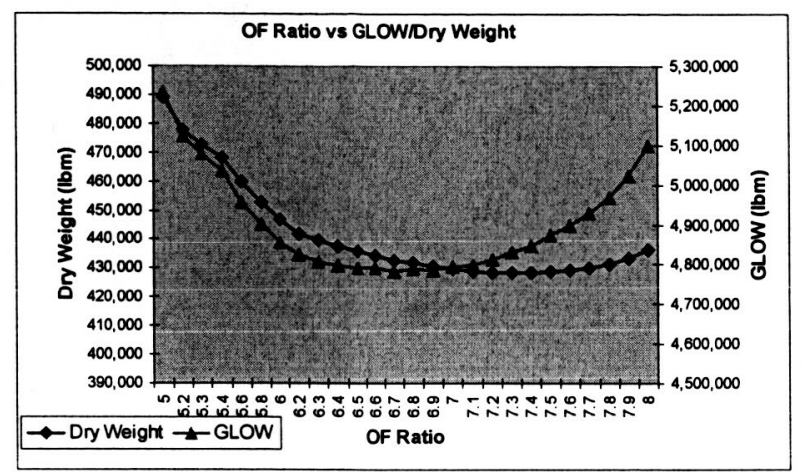

Figure 6 Effect of OF Ratio on Dry Weight

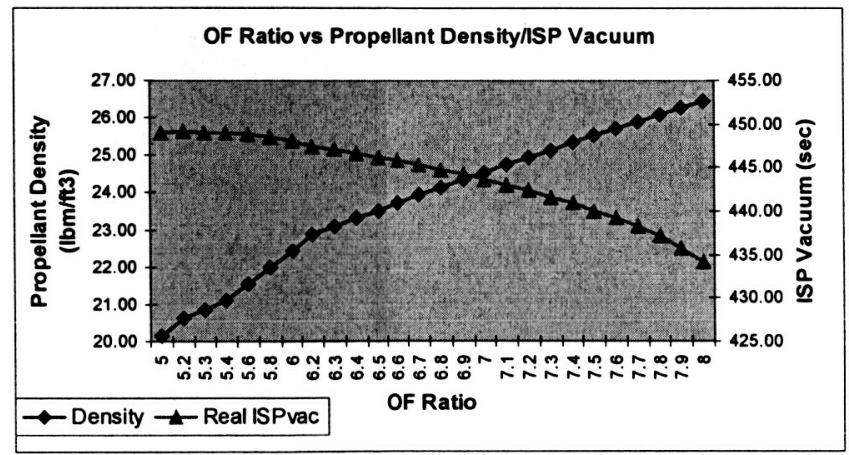

Figure 7 Effect of OF Ratio on Propellant Density

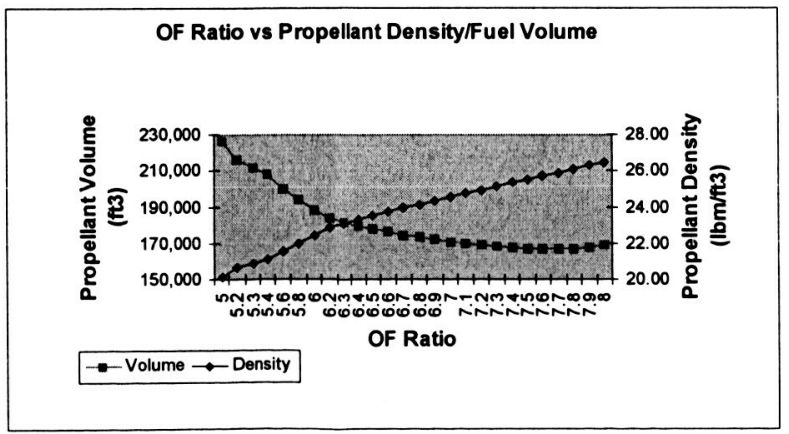

Figure 8 Effect of OF Ratio on Propellant Volume 


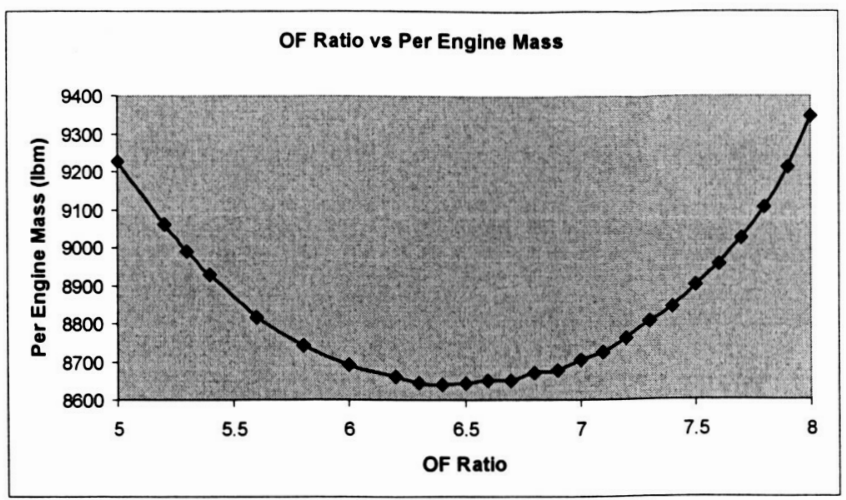

Figure 9 Effect of OF Ratio on Per Engine Mass

Figures 6 to 9 show the data plots for Dry Weight, GLOW, ISPvac, propellant volume, propellant weight, and engine mass versus OF Ratio. By examining Figure 6, the OF Ratio value for minimum GLOW has a range from approximately 6.2 to 7.1 where there is little impact on the GLOW. The data shows that when the OF Ratio is varied, the impact on the overall propellant weight is only $.75 \%($ OF Ratio $=6.2)$ to $.82 \%($ OF Ratio $=7.2)$ above the optimum OF Ratio. The results proves that for optimum GLOW, the best takeoff OF Ratio is in the range of 6.2 to 7.2 , if the system is a LOX/H2 fixed bell nozzle engine configuration. The results for minimum engine mass in Figure 9, is also consistent with this range of values. An OF Ratio in the range of 6.2 to 7.2 is best for the initial phase of the trajectory when the vehicle propellant mass is most important.

The maximum ISPvac is found at the OF Ratio of 5.2. These results are defined in Figure 7. An OF Ratio of 5.0 (ISPvac = 449.01) to $5.6($ ISPvac $=448.81)$ would be best for the later phase of the trajectory profile, when higher ISP is important. The ISP value changes only slightly over this range of values for OF Ratio. These OF Ratio results are consistent with the early plans for the SSME that called for an adjustable OF Ratio. The preliminary plans for the SSME included a value for OF Ratio equal to 6.5 for the initial takeoff, with the ratio being modified to 5.5 later in flight when the higher ISP was more important.

The Dry Weight and GLOW, for the range of Pc from 2000 to 3700 psi, increased only slightly above the minimum weights for the optimized Pc. The values for Dry Weight and GLOW versus Pc are shown in Figure 10. The optimum value for lowest Dry Weight and engine mass correlated to a Pc value near 2400 psi. The value for the lowest GLOW is near 2700 psi. Figure 11 illustrates that the ISPvac increases as Pc increases. This is a logical conclusion because higher pressures translate into higher ISP values. Figure 12 shows a significant increase in the engine mass as the Pc approaches 3700 psi or as the Pc value increases. The increased engine mass is due to the thickness and design of the thrust chamber, increased robust design for valves, ducts and 
lines needed at the higher pressures. The overall engine mass increase, due to higher pressures, is at such a rate that the improved weight of the nozzle mass does not offset the core engine mass increase.

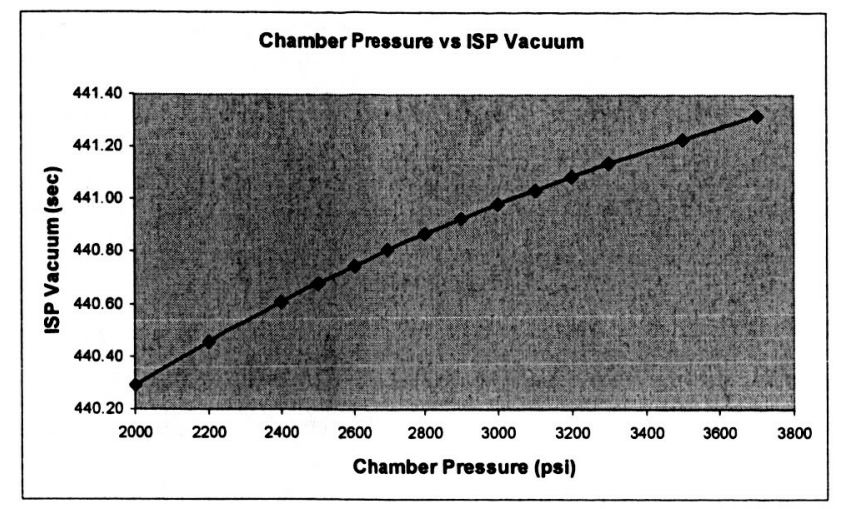

Figure 10 Effect of Chamber Pressure on Isp

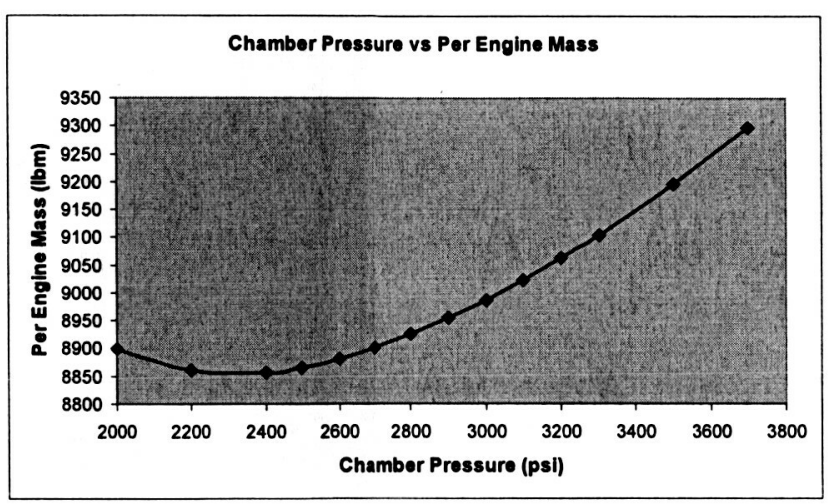

Figure 11 Effect of Chamber Pressure on Per Engine Mass

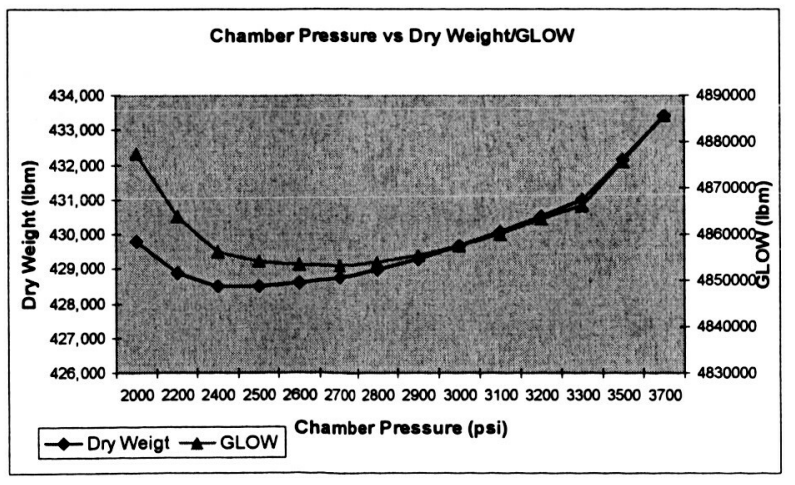

Figure 12 Effect of Chamber Pressure on Dry Weight 
The effects of Area Ratio on the vehicle system are shown in Figures 13 to 15 . The Dry Weight and GLOW change very little over the range of AR from 50 to 120. The incremental changes in vehicle Dry Weight, GLOW and ISPvac are shown in Figure 13. The optimum value for lowest Dry Weight correlated to an AR value in the range of 70 to 105 for a SSTO vehicle. Historically, the AR range for engines of multi-stage vehicles is from 45 to 60 . The AR range, for engines used to complete the entire trajectory from launch to orbit, is 69 to 85 . This is for the SSME (AR $=69$ to 77.5) and the Russian made RD-0120 (AR $=85$.

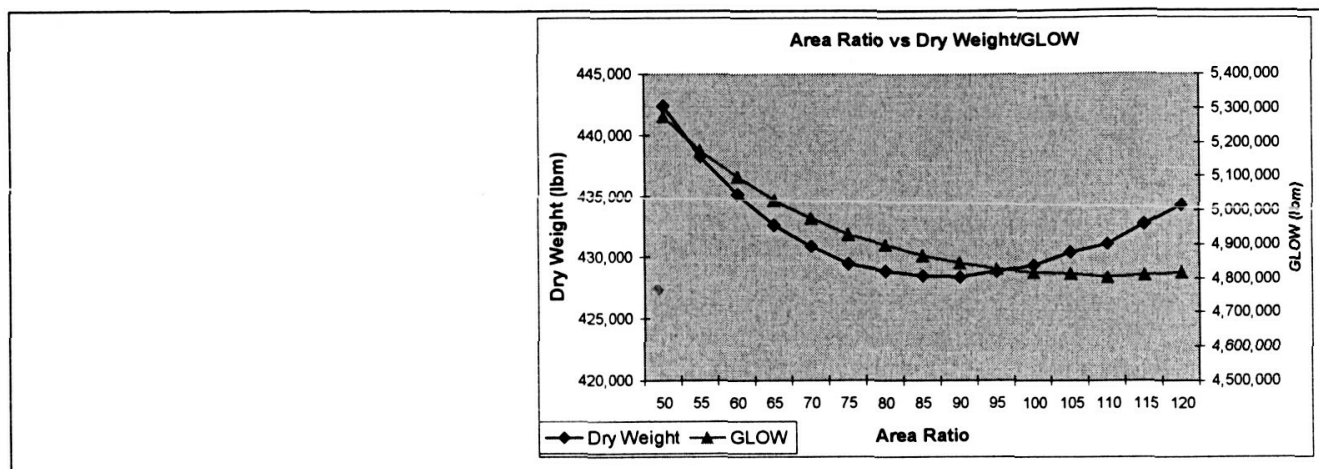

Figure 13 Effect of Area Ration on Dry Weight

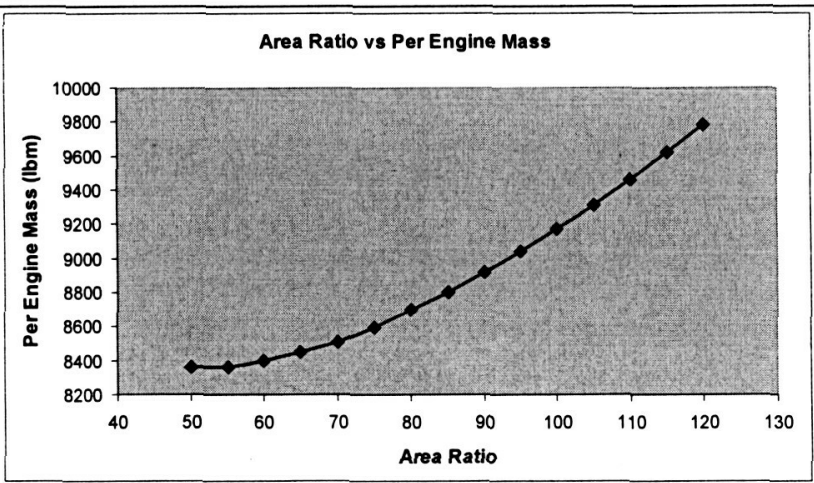

Figure 14 Effect of Area Ration on Per Engine Mass

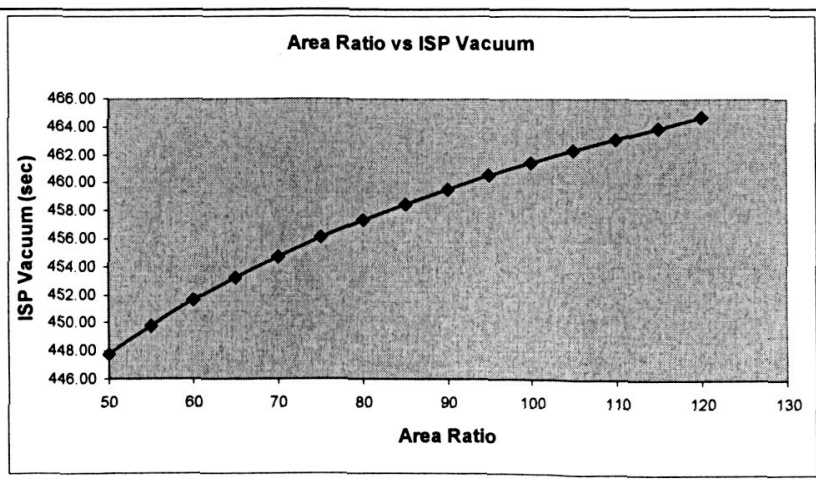

Figure 15 Effect of Area Ratio on Isp 
Figure 14 shows that the lower the AR the lower the engine mass. This is understandable due to the nozzle mass required for an $\mathrm{AR}$ equal to 50 versus an $\mathrm{AR}$ equal to 120. An AR of 50 corresponds to a much smaller exit area than the exit area for and engine with AR equal to higher values. The Figure 15 shows that ISPvac increases with the larger values for AR. This does not take into account the possibility of shocks in the nozzle due to over-expansion.

\section{A. SSME Comparison Case}

An SSME comparison case involves optimization where the total thrust level is in the SSME range of Tvac equal to 500,000 lbf. The optimized cases are shown below in Table 4. The Table relate the OF Ratio, AR, and Pc that corresponds the SSTO case where the SSME thrust level is used. The error percentages are given for the GLOW minimization using an engine Tvac of $500,000 \mathrm{lbf}$ as compared to the SSME design case.

The key comparison focuses on how the models optimum values for Pc, AR and OF Ratio differ from the actual design values for the SSME. The optimizer defined Pc is $2250 \mathrm{psi}$, where the SSME is designed to $3141 \mathrm{psi}$. This also manifest itself in a higher throat area due to the direct correlation between $\mathrm{Pc}$ and $\mathrm{A}^{*}$ in calculating the engine thrust level. The OF Ratio and AR are approximately $10 \%$ higher than the SSME case leading to the conclusion that the OF Ratio should be higher to achieve optimum GLOW minimization. The optimum engine OF Ratio is equal to 6.63. This value is consistent with the historical desire to have the shuttle system initial OF ratio set to 6.5 , instead of the 6.0 to which it is currently designed. The data results show that the engine should be designed to an area ratio of 86.7, which is consistent with the RD-0120's AR of 85.7.

Table 4, SSME Comparison Case 


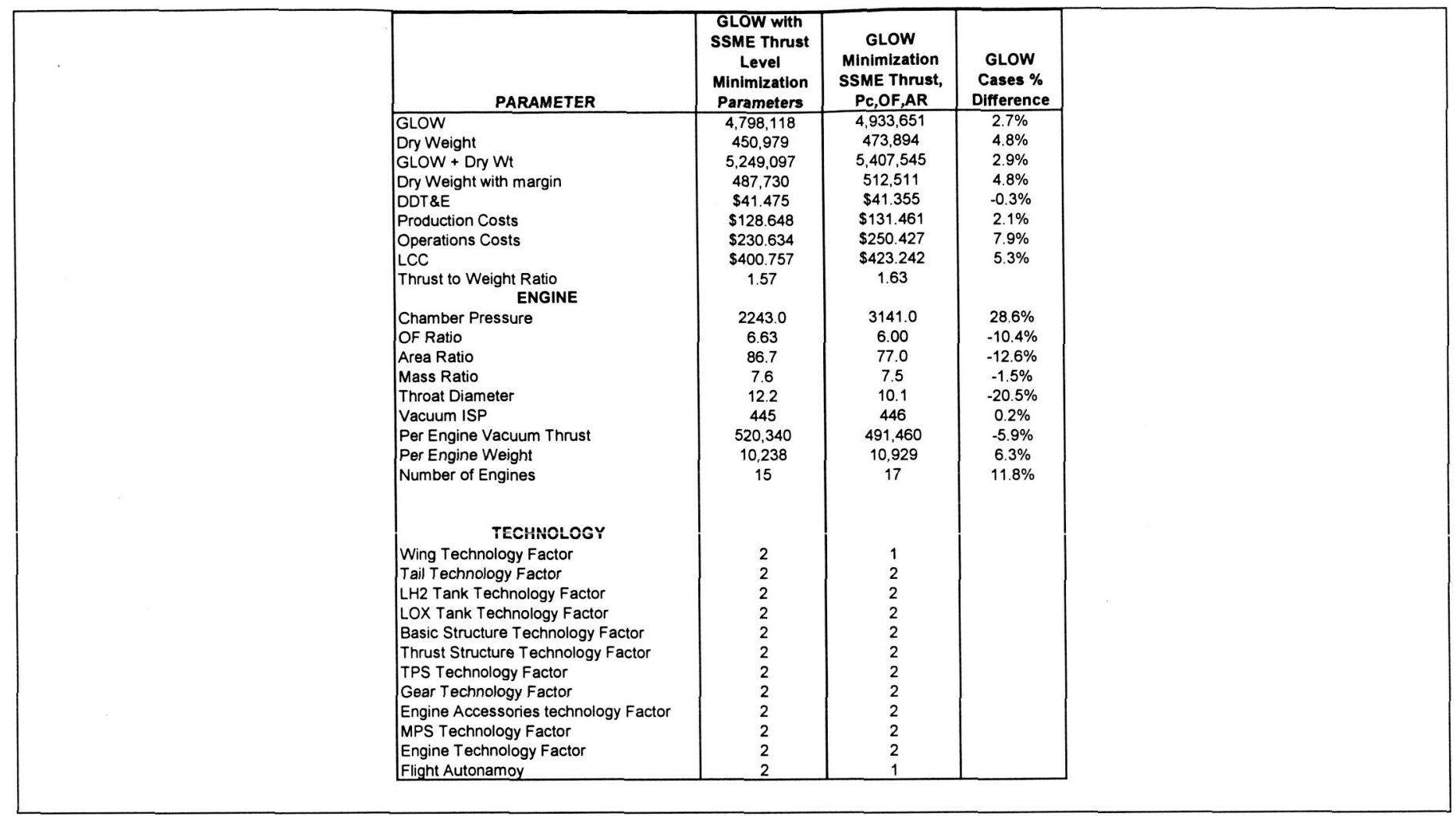

\section{B. Uncertainty}

There are two areas that uncertainty values apply in the model. The first is the DDTE cost uncertainty and the second is the uncertainty in the vehicle weight. The DDTE cost uncertainty is approached in two parts. The first part is the weight of the components that directly feed the NAFCOM regression curves and the other is the NAFCOM regression curves themselves. The component weight distributions will be used for both the uncertainty in weight and uncertainty in the DDTE cost since weight is the bases for the cost curves.

Weight regression curves were used to define the SE, $\mu$, and $\sigma$ for the vehicle components. This data is based upon historical data gathered from previously designed systems and is not linked to any of the NAFCOM Cost model data. The high and low values for the power series regression curve fits are define along with the corresponding Lognormal parameters necessary to formulate the distributions. The table data reflects the parameters used in the @Risk tools runs that exercise the Monte Carlo simulations.

The weight only portion of the overall DDTE cost uncertainty represents the first part of the total uncertainty in cost. This portion is dedicated to the uncertainty in the weight of the vehicle components. The second component of the uncertainty due to the regression errors is defined using the NAFCOM historical data/curve fits. The SEs for the NAFCOM regression fits are not 
shown herein but are combined with the data from the weight distribution curves to define a total uncertainty distribution for the projected cost of the vehicle.

The uncertainty in the DDTE cost is computed by talking each of the component cost equations and multiplying the weight by the uncertainty in weight and the overall cost equation by the uncertainty in the NAFCOM regression curve. The equations (1.11) to (1.13) below are an explanation of the DDTE cost uncertainty and shows how the Lognormal distributions for weight uncertainty (Wtunc) and NAFCOM uncertainty (NAFCOMunc) will be exercised as a multiplier of the terms in order to determine the total uncertainty in the cost number for a specified component (Wing, Tail, Tanks, etc.),

$$
\begin{aligned}
& \text { Wing }_{\text {unc }}=C I F * \text { CompFactor } * A * W t_{\text {wing }} B \\
& W t_{\text {wing }}=W t^{*} W t_{\text {unc }}
\end{aligned}
$$

and

$$
A^{*} W t_{\text {wing }}{ }^{B}=A^{*} W^{B} * N A F C O M_{u n c}
$$

The total DDTE cost uncertainty was defined by operating 10,000 Monte Carlo runs in @Risk using NAFCOM Lognormal distributions that result from the SE's of the components that make up the vehicle. The total uncertainty is defined in the range shown here in Figure 16. The sensitivity of the individual components that make up the total uncertainty is shown in Figure 17. The maximum and minimum values for uncertainty are presented here based upon the 95 th percentile case. The absolute minimum and maximum values are decidedly outside the bounds of acceptable engineering estimates. This is the benefit of using the 95th percentile case to describe the bounds of the DDTE cost uncertainty. The data defines the lower and upper values of uncertainty at $-45 \%$ to $76 \%$ of the mean value of DDTE cost. The results show that there is a significant level of uncertainty in the cost based upon the uncertainty in component weights and upon the NAFCOM uncertainty.

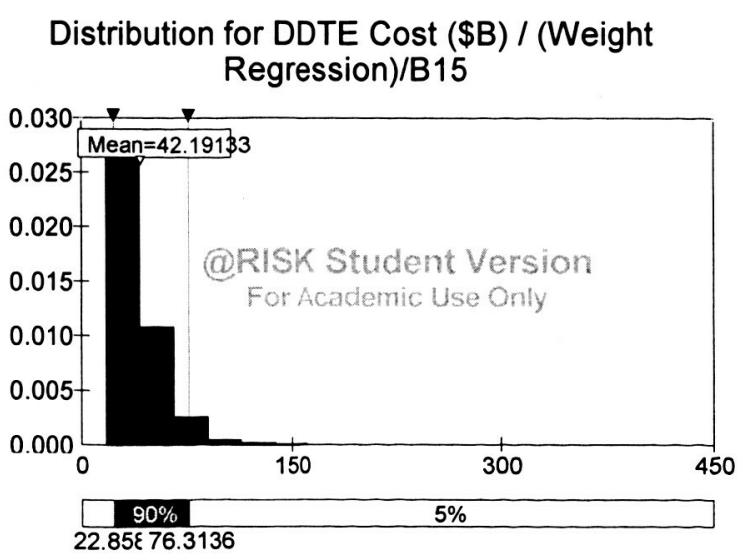

Figure 16 DDTE Cost Distribution 
Regression Sensitivity for DDTE Cost (\$B) /

(Weight Regr...

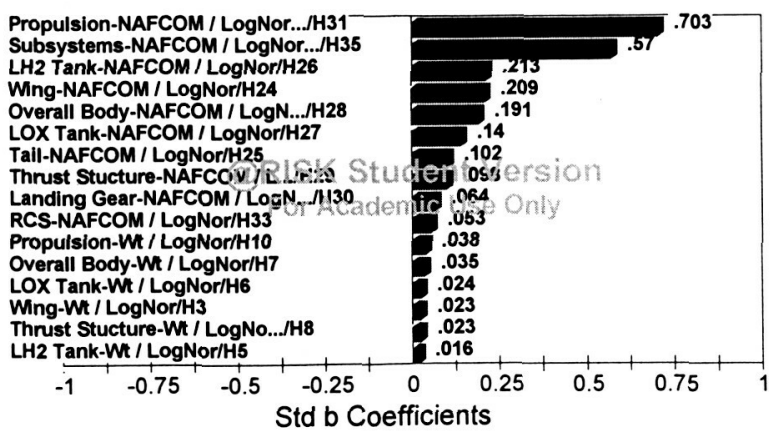

Figure 17 Regression Senstivity for DDTE Cost

In a similar manner as defined in the previous section, the distributions are developed for each of the vehicle components (wings, tail, tanks, etc.). The SE is calculated using power series regression curves. Talking each of the component weights and multiplying the weight by the uncertainty in weight computes the uncertainty vehicle weight. The equation (1.14) below is an example of the component weight uncertainty and will be used in order to determine the total uncertainty in the weight for a specified component (Wing, Tail, Tanks, etc.),

$$
\text { Wing }_{\text {unc }}=W t_{\text {wing }} * W t_{\text {unc }} \text {. }
$$

The total uncertainty is defined in the range shown here in Figure 18 for the Dry Weight and Figure 19 for the GLOW. The maximum and minimum values for uncertainty are presented here based upon the 95 th percentile case. The absolute minimum and maximum values are decidedly outside the bounds of acceptable engineering estimates. This is the benefit of using the 95 th percentile case to describe the bounds of the uncertainty. The data in Table defines and lower and upper values of uncertainty for Dry Weight and GLOW. The results show that there is a significant level of uncertainty based upon the uncertainty in component weights. 


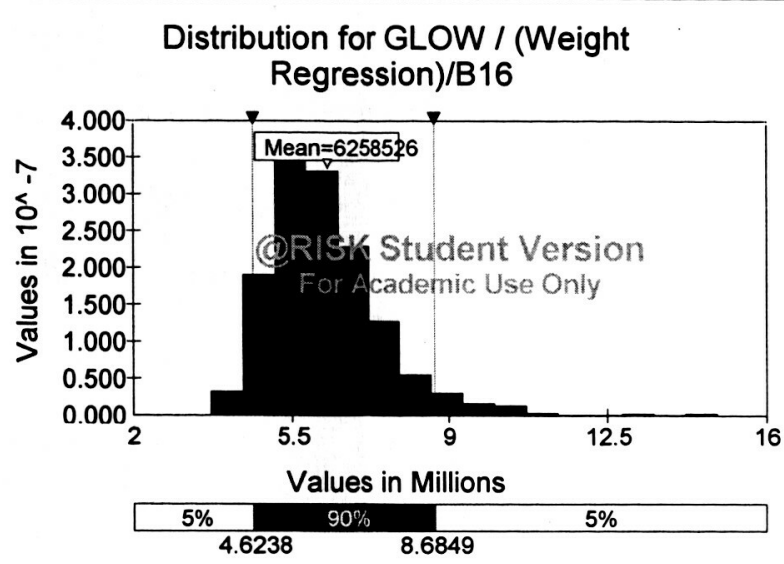

Figure 18 Distribution for GLOW/Weight

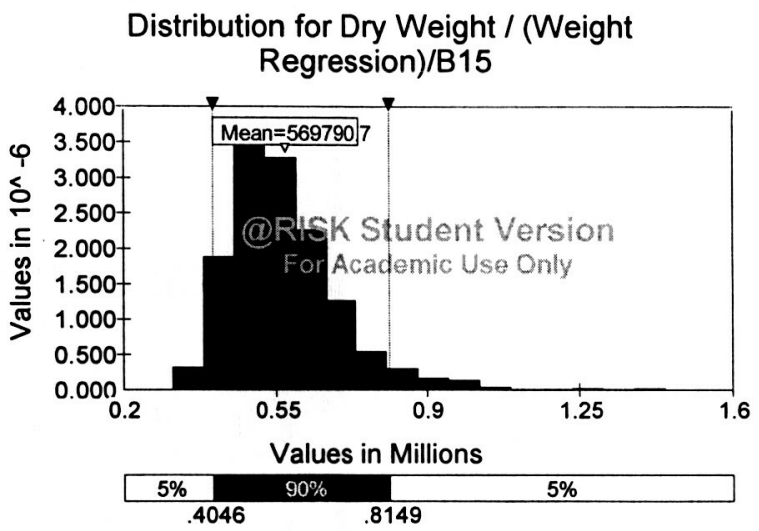

Figure 19 Distribution for Dry Weight

\section{Conclusions}

Using the Cequel code for thermo-chemical outputs, by inputting Pc, AR and OF Ratio, provided a methodology that has not been used in previously designed tools attempting to optimize a closed loop system. The Cequel output and input structure designed in the linkages proved that a true closed loop system produces the results in a significantly more efficient way. The alternatives to integrating thermo-chemical results into the model are to use databases and extrapolate the results for a given input condition.

A key aspect of the model was the method to determine engine mass based upon parameters of the system or propulsion variables produced in the design process. Design synthesis of the proposed propulsion parameters and the goodness of the engine mass models led to the optimum design being chosen that closely matches the theoretical optimum vehicle. The engine 
mass modeling approach is open to updates based upon any new and/or improved methods to estimate the mass using design parameters. The ability to have the engine mass, parameters used to determine engine mass, and the vehicle weight and cost integrated together in an optimized fashion is the essence of CLO. The model outputs are only as good as the data being used to formulate the model. This point must not be overlooked and an emphasis must be made toward higher fidelity modeling in future efforts where CLO is attempted.

The integrated model output defined that the GLOW and Dry Weight was $67 \%$ higher for the DDTE cost minimization case when compared to the weights determined from the minimization of GLOW case. This is driven by the nature of the parameters tendency toward making the optimized value in each case as small as possible. In a likewise manner, The DDTE cost was $53 \%$ higher using the GLOW minimization case when compared to the case for minimization of the DDTE cost. Again, this was an expected result due to the higher cost for the case where weight is being optimized. These variables provide valuable insight into program management trades where cost and vehicle weight are involved.

The uncertainty of the model results are defined using the Lognormal distributions based upon the power series regression curves and SE values for each component weight and SE values for the NAFCOM regression curves. By using the Lognormal distributions, the data being used in the Monte Carlo runs was significantly more accurate than a Triangular distribution or Normal distribution. This was true because the Lognormal curves represent the log space distribution of the power curve data that is plotted on logarithmic scale axis. The model output for DDTE cost uncertainty was approximately $76 \%$ for the 95th percentile upper limit. According to feedback from NASA and Academia experts, this closely matches the $80 \%$ value used for program planning purposes. The Dry Weight uncertainty output reflected a 95 th percentile uncertainty upper range that was $+90.2 \%$ above the model output for Dry Weight minimization $(428,123 \mathrm{lbs})$. Likewise, the GLOW uncertainty output had a value of $81.3 \%$ above the model output for GLOW minimization $(4,635,904 \mathrm{lbs})$.

The model output for the core propulsion parameters was a significant result of the optimization effort. The chamber pressure for the best GLOW and Dry Weight are 2712.8 and 2464.9 psi respectively that compares to 2994 psi for the Block II SSME. The likelihood of these optimized values being the true optimized value can be clarified by further efforts in engine mass studies and engine reliability studies. Engine reliability is an important aspect of the engine modeling approach but was not included in this optimization approach.

The AR for optimum engine design, for the minimization of Dry Weight [case 2], was 86.7 and is almost identical to the Russian RD-0120 engine AR value of 85.7. These results, along with the fact of close correlation with the SSME and RD-0120, show that the model's optimum value for AR was close to the true optimum value. The conclusion can be drawn that the AR for 
SSTO systems should slightly be higher than the SSME and significantly higher than the 1st stage engines used in expendable launch vehicles.

The OF Ratio was the most sensitive component of the propulsion parameters due to its overwhelming impact on the propellant weight and tank volume. This was due to propellant mass, that was significant to the GLOW, and the propellant volume, that was significant to the Dry Weight. The optimum OF Ratio for GLOW minimization was found by considering the GLOW for the launch vehicle. The GLOW was most impacted by the propellant weight. Another driving force behind the OF Ratio was that the maximum specific impulse occurs at the ratio of 5.2. Using in these driving factors, the optimum OF Ratio for fuel weight was defined 6.87 for the GLOW optimization case involving the engine with Tvac equal to 800,000 . The optimum OF Ratio for the SSME thrust level of 500,000 lbf was defined at 6.63 .

The importance of the TFs and CIFs cannot be underestimated in determining the optimum system with respect to cost and weight. The base technology represented by the Boolean code one (1) does not improve the optimum system weight but does make the system cheaper to design and test. The technology improvements and weight savings represented by Boolean codes two (2) and three (3) are significant weight reducers but do increase the overall DDTE cost. The interesting result the variability amongst the TFs for the optimum production, operations and life cycle cost cases. The CIFs have no direct impact on these cost cases. Their only influence is with the input weights and DDTE cost provided by the model components. A comparison of the optimization cases show that system tradeoffs can be made to improve the cost of the system at the expense of vehicle weight. This was also true for the reverse. The improvement in overall vehicle weight resulted in significant increases in the cost of the system. The key component of trading cost and weigh proved to be the technology and cost influence factors resulting from the inclusion of new technologies. The engine design parameters has some impact on the overall system optimization but the effects are minimal in comparison the technology factors.

\section{Acknowledgments}

I also want to acknowledge the advice from Joe Leahy/MSFC. Joe shared his insight into the P-Star tool being developed and used by the NASA MSFC Space Transportation Directorate and was always available for questions and suggestions. I also want to thank Dr. Christian Smart/SAIC-Huntsville for his help in understanding the NAFCOM cost uncertainty approaches and his sharing insights on lognormal distributions.

\section{References}

[1] Haefeli, R., Littler, E., Hurley, J., Winter, M., Martin Marietta Corporation, “Technology Requirements for Advanced EarthOrbital Transportation System”, NASA Contractor Report, NASA CR-2866, October 1977. 
[2] Palisade Corporation Software, @ RISK ${ }^{\mathrm{TM}}$ Risk Analysis and Simulation Software, Evolver Tool Software, 'Decision Tools $4.5^{\prime}, 2002$.

[3] Software and Engineering Associates, Inc., 'Cequel', 2001-2004.

[4] Leahy, J., "P-STAR: A Propulsion Sizing, Thermal Analysis and Weight Relationship Model", NASA George C. Marshall Space Flight Center, AL, 2003.

[5] Sutton, G., Rocket Propulsion Elements An Introduction to the Engineering of Rockets, $6^{\text {th }}$ ed., John Wiley \& Sons, New York, 1992.

[6] Paulson, E., Burkhardt, W., Mysko, S., Jenkins, J., "Simplified Liquid Rocket Engine Performance and Weight Model”, JANNAF CS'APS/PSHS \& MSS, Cólorado Springs, CO, pages 4-6́, Sepiember 1-5, 2003.

[7] Wilhite, A., NASA Langley Research Center, "Engine Weight Study", 2004.

[8] Wells, N., "Bell Parametrics", Rocketdyne a Division of North American Rockwell Corporation, Model ILRV, pages 4-33, December 1969.

[9] Wilhite, A., Gholston, S., Farrington, P., Swain, J., University of Alabama in Huntsville, "Evaluating Technology Impacts on Mission Success of Future Launch Vehicles", IAF-01-V.4.04, 52 ${ }^{\text {nd }}$ International Astronautical Congress, Toulouse, France, October 2001 .

[10] Smart, C., "NAFCOM Cost Risk Module", The NASA Engineering Cost Group and Science Application International Corporation, Huntsville, AL., pages 2-8, 2004.

Words $\quad 7,700$

Figures $19 \times 200 \quad 3,800$

Tables $4 \times 200=\quad 800$

$\begin{array}{ll}\text { TOTAL } & 12,300\end{array}$ 